\title{
Phosphorus Remains Exciting!
}

\author{
Arno Pfitzner*
}

\section{Keywords:}

element modifications - phosphorus .

structure elucidation
Today, the search for new element modifications is basically characterized by work under extreme conditions, in particular, high pressures and very often high temperatures. This situation is not surprising since the discovery of new elements and their characterization under normal conditions is more a topic of chemical-historical literature. Despite this, surprises are still found in the area of the main-group elements, such as the discovery of the fullerenes for which Curl, Kroto, and Smalley were awarded the Nobel Prize in 1996. In addition to carbon, there is another element for which new modifications can be envisaged when kinetic effects are considered, and whose phase diagram still has unexplained features: phosphorus. Initially this notion appears surprising because the element has been known for about 350 years, but when the numerous possibilities for phosphorus atoms to bond with one another are taken into account, the idea seems quite plausible.

In 1995 Böcker and Häser investigated theoretically and systematically the energetic relationships between the many plausible bonding patterns of phosphorus. ${ }^{[1]}$ This work showed that, for example, a whole series of energetically equivalent possibilities exists for the formation of phosphorus polymers. Presumably this situation also explains the difficulties encountered clarifying the structures of different forms of the so-called red phosphorus. As well as the

[*] Prof. Dr. A. Pfitzner

Institut für Anorganische Chemie

Universität Regensburg

93040 Regensburg (Germany)

Fax: (+49) 941-943-4983

E-mail: arno.pfitzner@chemie.uni-regensburg.de multiatom polymers, there is also tetrahedral $\mathrm{P}_{4}$, which is a very small phosphorus molecule that is familiar to every chemist.

The discovery of phosphorus lies far in the past but now the time at last appears to be ripe for a whole series of new experimental findings. Thus Ruck et al. ${ }^{[2]}$ have reported the elucidation of the crystal structure of socalled fibrous phosphorus, a form that had been postulated by Thurn und Krebs as early as $1969 .{ }^{[3]}$ Apparently, depending upon the crystallization conditions, the polymeric phosphorus tubes with the familiar pentagonal cross-section are either linked crosswise with one another (Hittorf's or violet phosphorus) or parallel so that double tubes are formed (fibrous phosphorus). According to the current results of Ruck et al., Hittorf's phosphorus is formed from amorphous red phosphorus either from lead melts or by heating with small amounts of iodine. In contrast, the fibrous modification was not obtained from lead; crystals in detectable amounts are formed best in a temperature gradient of approximately $10 \mathrm{~K}$ at about $580 \mathrm{~K}$. Ruck et al. conclude from their observations that crystallization of the tubes on the wall of the quartz glass ampoule supports the parallel orientation of the tubes. They were able to determine the crystal structure of the fibrous modification of phosphorus by X-ray diffraction on a single crystal (Figure 1) and demonstrate the clear difference from Hittorf's phosphorus by high-resolution electron diffraction imaging.

The extensive theoretical investigations in ref. [2] showed that unlike Hittorf's phosphorus, in the fibrous form the $\mathrm{P}-\mathrm{P}$ bond between the strands has no $\pi$-bonding component. At this posi-
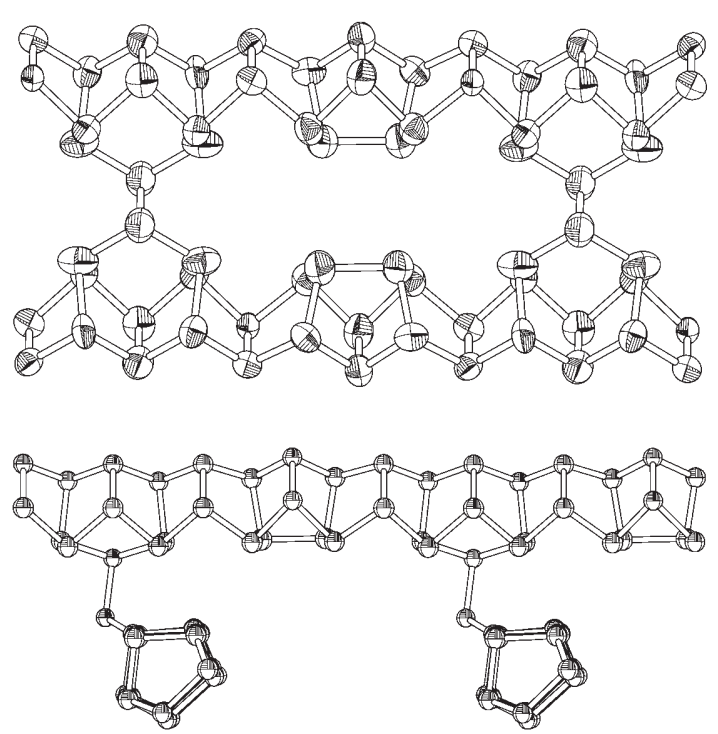

Figure 1. Linkage of the phosphorus strands in fibrous phosphorus (top; data from ref. [2]) and Hittorf's phosphorus (bottom; data from ref. [3]).

tion the fibrous modification has a trans conformation, whereas the linkage in Hittorf's phosphorus can be regarded as a gauche conformation. In this way the significantly different bond lengths at this position can be explained very well. In contrast to earlier assumptions ${ }^{[3]}$ the density of both forms is the same. It may be concluded from this result that the synthesis conditions are solely responsible for the formation of the one or the other modification, and not, for instance, different thermodynamic stabilities. This situation also explains the simultaneous formation of Hittorf's and fibrous phosphorus from the gas phase. Calculations confirm the energetic equivalence of both allotropes.

According to theoretical investigations by Häser it can be assumed that in addition to these two differently linked tubes of phosphorus with pentagonal cross section, the polymers with the 


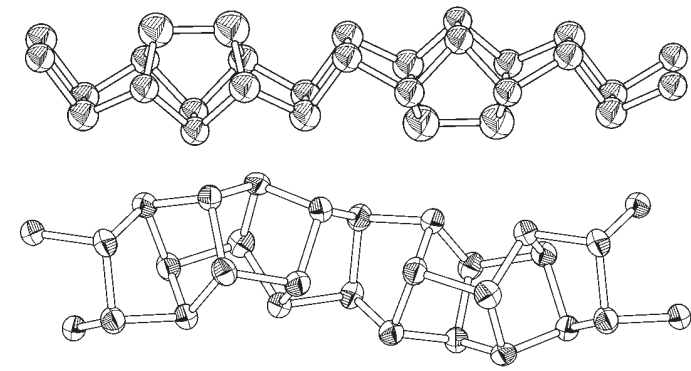

Figure 2. $\left[\mathrm{P}_{12}\right]$ polymers which according to data in ref. [1] represent energetically equivalent (bottom) or only slightly less favorable alternatives (top) to the pentagonal, linked tubes (from ref. [4]).

translational unit $\left[\mathrm{P}_{12}\right]^{[4]}$ (Figure 2) isolated recently from their copper iodide adducts are also similarly energetically favorable. To date, however, it has not been possible to obtain them in crystalline form, instead, as a result of the synthesis conditions and possibly their poorly packable structures they occur as amorphous, nanoscale rods. Unlike the crystalline modifications that are obtained at relatively high temperatures, the nanoscale, unlinked phosphorus rods are formed at room temperature.
Thus within just one year a whole series of alternatives to Hittorf's phosphorus has been synthesized and characterized. By consideration of the synthetic and investigational methods it becomes clear that only by the combination of the different methods available today (e.g. high-resolution transmission electron microscopy, solid-state NMR spectroscopy, and DFT calculations) was a satisfactory characterization possible. The time is therefore ripe finally to fill in the remaining white patches on the phosphorus phase diagram.

New work on crystalline white phosphorus $\left(\mathrm{P}_{4}\right)$ has also been performed. At the beginning of the year, the crystal structure of the $\gamma$-phase of $\mathrm{P}_{4}$ was determined by X-ray diffraction on powder samples. ${ }^{[5]}$ To obtain this phase the phase-transformation behavior of $\mathrm{P}_{4}$ during rapid temperature change was used. The structure was solved from powder data with the aid of modern methods (simulated annealing and subsequent Rietveld refinement). A more accurate analysis of $\gamma-\mathrm{P}_{4}$ with theoretical methods shows that the lone electron pair on the phosphorus atoms influences the crystal structure. In this way the deviations of the structure from the an expected "ideal structure" can be explained very well by an optimization of the van der Waals forces between tetrahedral molecules. ${ }^{[5]}$

Published online: December 28, 2005

[1] S. Böcker, M. Häser, Z. Anorg. Allg. Chem. 1995, 621, 258.

[2] M. Ruck, D. Hoppe, B. Wahl, P. Simon, Y. Wang, G. Seifert, Angew. Chem. 2005, 117, 7788; Angew. Chem. Int. Ed. 2005, $44,7616$.

[3] H. Thurn, H. Krebs, Acta Crystallogr. Sect. B 1969, 25, 125.

[4] A. Pfitzner, M. F. Bräu, J. Zweck, G. Brunklaus, H. Eckert, Angew. Chem. 2004, 116, 4324; Angew. Chem. Int. Ed. 2004, 43, 4228.

[5] H. Okudera, R. E. Dinnebier, A. Simon, Z. Kristallogr. 2005, 220, 259. 